\title{
Role of Cardiac MR Imaging in Cardiomyopathies
}

\author{
Christopher M. Kramer \\ Cardiovascular Imaging Center, University of Virginia Health System, Charlottesville, Virginia
}

\begin{abstract}
Cardiac MR imaging has made major inroads in the new millennium in the diagnosis and assessment of prognosis for patients with cardiomyopathies. Imaging of left and right ventricular structure and function and tissue characterization with late gadolinium enhancement (LGE) as well as T1 and T2 mapping enable accurate diagnosis of the underlying etiology. In the setting of coronary artery disease, either transmurality of LGE or contractile reserve in response to dobutamine can assess the likelihood of recovery of function after revascularization. The presence of scar reduces the likelihood of a response to medical therapy and to cardiac resynchronization therapy in heart failure. The presence and extent of LGE relate to overall cardiovascular outcome in cardiomyopathies. A major role for cardiac MR imaging in cardiomyopathies is to identify myocardial scar for diagnostic and prognostic purposes.
\end{abstract}

Key Words: cardiac MR imaging; coronary artery disease; late gadolinium enhancement; ischemic cardiomyopathy; nonischemic cardiomyopathy; infiltrative cardiomyopathy

J Nucl Med 2015; 56:39S-45S

DOI: 10.2967/jnumed.114.142729

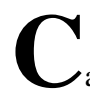

ardiac MR imaging (CMR) is considered the gold standard for measurement of the structure and function of the left ventricle (LV) and the right ventricle (RV). Cine imaging is used to cover the LV in the short axis from the apex to the base, essentially creating a 3-dimensional volumetric structure for analysis. In the 1990s, gradient-echo cine imaging was the standard, but this technique was replaced at the turn of the millennium by steady-state freeprecession cine imaging, which offers a higher contrast-to-noise ratio between the dark myocardium and the bright blood pool (1). Multiple studies have demonstrated the accuracy and reproducibility of CMR for measuring LV volumes, ejection fraction (EF), and regional function (2). Measurements made with this 3-dimensional dataset do not require geometric assumptions and are therefore less prone to error than 2-dimensional methods, such as 2-dimensional echocardiography, in ventricles deformed by myocardial infarction or cardiomyopathies. Interstudy and interscan reproducibility is high, allowing for reduced sample sizes in clinical trials of heart failure $(3,4)$. Techniques such as myocardial tissue tagging (5) or cine displacement-encoded stimulated-echo imaging (6) allow more detailed analysis of regional myocardial motion and

\footnotetext{
Received Nov. 13, 2014; revision accepted Dec. 26, 2014.

For correspondence or reprints contact: Christopher M. Kramer, Cardiovascular Imaging Center, University of Virginia Health System, P.O. Box 800170, Lee St., Charlottesville, VA 22908-0001.

E-mail: ckramer@virginia.edu

COPYRIGHT (c) 2015 by the Society of Nuclear Medicine and Molecular Imaging, Inc.
}

deformation than cine imaging and have been applied to studies of myocardial infarction (7) and hypertrophic cardiomyopathy (HCM) (8).

Late gadolinium enhancement (LGE) refers to the discrimination of regions of scar, necrosis, or inflammation from normal tissue by the prolonged retention of gadolinium-based contrast agents. Since the mid-1980s, investigators have appreciated T1 shortening (increased enhancement) in regions of infarction after gadolinium administration (9-11). However, these early imaging techniques were limited by long acquisition times, artifacts, and insufficient contrast between normal and abnormal regions. With faster approaches and the implementation of an inversion recovery pulse sequence with the inversion time set to null normal myocardium, the signal difference between normal and infarcted segments has been increased by $500 \%-1,000 \%$ (12). Using this approach, Kim et al. demonstrated that the spatial extent of LGE seen on CMR closely mirrored the distribution of myocyte necrosis in the early period after infarction and that of collagenous scar at $8 \mathrm{wk}(R=0.97, P<0.001)(13)$, whereas in regions of the heart subjected to reversible injury, the retention of contrast did not occur (Fig. 1) (14). LGE accurately delineates infarction, as defined by histology, at various time points after injury (15). LGE is not unique to infarct scar and can demonstrate any cause of fibrosis or infiltration in cardiomyopathies.

T2-weighted imaging can be used to demonstrate myocardial edema or injury. An understanding of the time course of edema is key to the accurate interpretation of T2-weighted imaging. Using a canine model of myocardial infarction, Abdel-Aty et al. noted that the onset of edema on CMR first became apparent $28 \pm 4 \mathrm{~min}$ after experimental coronary artery occlusion and well before the appearance of LGE or troponin elevation, signifying that CMR can visualize edema before the onset of irreversible myocardial injury (Fig. 2) (16). Microsphere analysis in a canine model of reperfusion after acute myocardial infarction suggested that T2weighted imaging performed $2 \mathrm{~d}$ after myocardial infarction can be used to delineate the area at risk (17). Increased T2 signal intensity can be seen in any cause of myocardial injury, such as acute myocarditis (18). Older T2-weighted imaging approaches were prone to artifacts; more recently, T2 mapping has been used with CMR protocols to overcome these limitations (19).

$\mathrm{T} 1$ of the myocardium can be measured by mapping with a modified Look-Locker inversion recovery sequence (20) and shorter versions of this approach (21). Native or precontrast T1 can be measured before contrast infusion, and then postcontrast T1 measures can be used to calculate the extracellular volume fraction in the myocardium (22). These techniques are being applied to help in the differential diagnosis of cardiomyopathies, as some have characteristically high native T1 values as well as high extracellular volume fractions. Native T1 is especially useful in patients with concomitant renal disease, as patients with stage 4 or 5 chronic kidney disease are not candidates for 


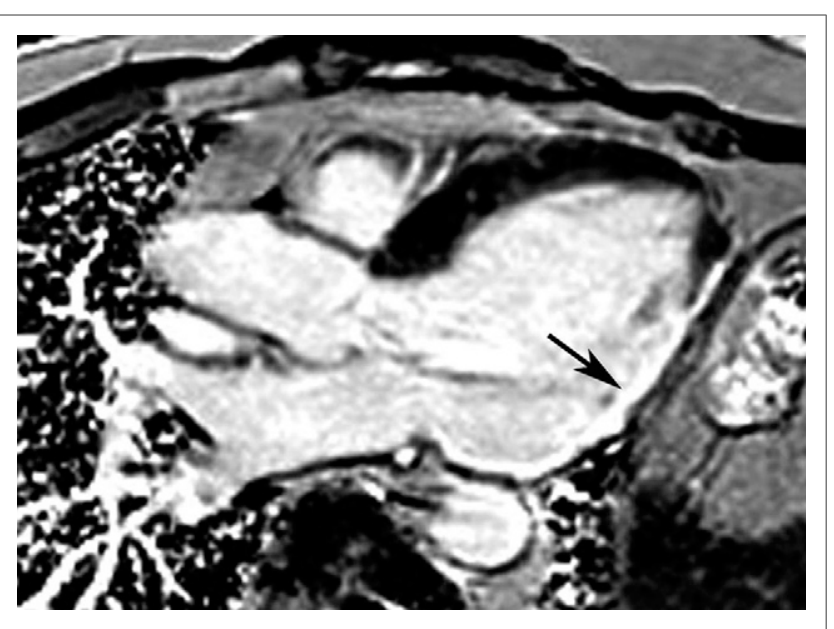

FIGURE 1. Three-chamber long-axis phase-sensitive inversion recovery LGE image in patient with heart failure and CAD. Transmural LGE can be seen in inferolateral wall (arrow).

receiving gadolinium because of concerns about nephrogenic systemic fibrosis.

\section{ESTABLISHING ETIOLOGY OF CARDIOMYOPATHY}

The first step in the evaluation of the patient with new-onset heart failure is to evaluate the underlying etiology and, importantly, to exclude ischemic heart disease as a potentially reversible cause. The presence of LGE in a coronary distribution can support the diagnosis of underlying coronary artery disease (CAD), but its absence does not rule it out, as patients with extensive hibernating myocardium may have no LGE (Fig. 3) (23). In a study by Soriano et al. of 71 patients with new-onset heart failure and systolic dysfunction, the sensitivity of the infarct pattern of LGE for ischemic cardiomyopathy-as defined by the presence of obstructive CAD—was $81 \%$, whereas the specificity was $91 \%$ (23). Patients without obstructive CAD may have evidence of LGE in an infarct pattern due to thrombotic occlusion of a nonobstructed artery, embolization, or spontaneous coronary dissection and thereby may be misclassified. This finding was noted in $13 \%$ of 63 patients who had the diagnosis of dilated cardiomyopathy (DCM) with chronic heart failure (24). CT coronary angiography may be an excellent way of noninvasively ruling CAD in or out in the setting of new- or recent-onset heart failure, as shown in a study with a combination of CT coronary angiography and LGE determined by CMR as a gold standard for establishing the underlying etiology (25).

\section{ISCHEMIC CARDIOMYOPATHY}

In addition to identifying $\mathrm{CAD}$ as the underlying etiology, $\mathrm{CMR}$ can define appropriate therapy as well. A response to $\beta$ blockade can be predicted by the presence and extent of LGE. Bello et al. demonstrated an inverse relationship between the extent of LGE and the likelihood of contractile improvement with 6 mo of $\beta$ blockade (26). Fifty-six percent of segments with no scar demonstrated improved function, whereas only $3 \%$ of segments with greater than $75 \%$ transmural scar demonstrated improvement (26).

CMR plays an important role in the assessment of myocardial viability for the selection of patients for revascularization. An LV wall thickness of less than $5.5 \mathrm{~mm}$ at the end of diastole on rest cine CMR is generally considered a marker of nonviable myocardium (27).
One study, however, demonstrated that in a minority of patients who had wall thinning without a substantial LGE burden (4\% of 1,055 patients with $\mathrm{CAD}$ ), contractile recovery could occur after revascularization (28). Nontransmural infarcts typically do not result in severe end-diastolic wall thinning. The extent of LGE is a useful marker of the likelihood of functional recovery with revascularization. In a landmark study, Kim et al. studied 50 patients who had LV dysfunction and were evaluated with contrast-enhanced CMR before surgical or percutaneous revascularization (29). LGE was noted in $80 \%$ of the patients. There was an inverse relationship between the transmural extent of LGE and functional recovery with revascularization (Fig. 4). Segments with greater than 50\% transmural LGE were nonviable, whereas segments with no LGE had an approximately $80 \%$ likelihood of functional recovery. Similar findings were noted in a study of 52 patients undergoing coronary artery bypass surgery; in that study, $36 \%$ of segments developed new areas of LGE after surgery (30).

Cine imaging of long- and short-axis views of the LV after the administration of low-dose dobutamine $(10 \mu \mathrm{g} / \mathrm{kg} / \mathrm{min})$ can be used to assess contractile reserve and to predict areas of functional recovery with revascularization $(27,31)$. Bove et al. studied patients before and after coronary artery bypass surgery and divided segments into those with no LGE and those with $1 \%-25 \%, 26 \%-50 \%$, or greater than $50 \%$ transmural LGE (31). Dysfunctional segments were defined as those having wall thickening of less than 27\% (2 SDs less than normal myocardium). After revascularization with coronary artery bypass surgery, the improvement in wall thickening in segments with $1 \%-50 \%$ transmural enhancement was $22 \% \pm 4 \%$ for dobutamine-responsive segments; this improvement was significantly higher than that for non-dobutamine-responsive segments $(9 \% \pm 4 \%)$. These findings suggested that contractile reserve was more predictive of functional recovery than infarct transmurality in infarcts with 1\%-50\% transmural LGE. In a complementary study, 29 patients with ischemic cardiomyopathy and a severely reduced EF underwent both LGE and low-dose dobutamine cine imaging (32). On the basis of the area under the receiver operating characteristic curve, contractile reserve with low-dose dobutamine was superior to the presence of LGE in predicting the recovery of LV function, especially when myocardial segments had less than $75 \%$ transmural LGE. A recent metaanalysis of 24 studies involving

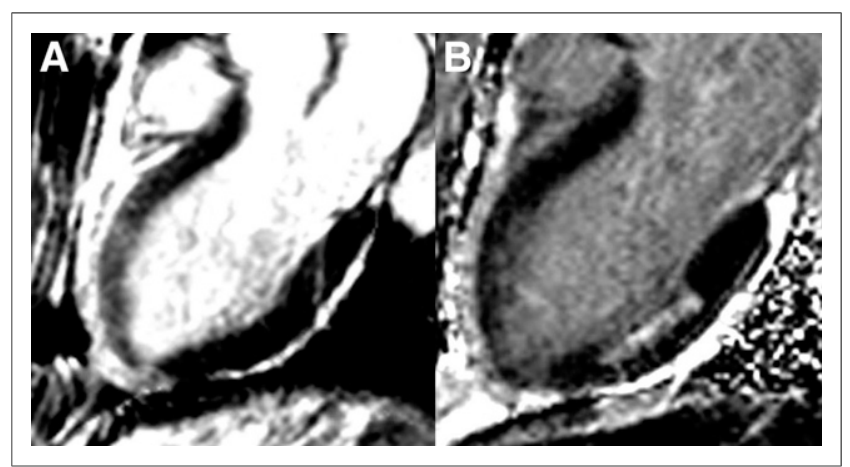

FIGURE 2. (A) Three-chamber long-axis bright-blood T2-weighted image in patient with spontaneous left anterior descending artery dissection. Bright signal in anterior wall is consistent with edema. (B) Three-chamber long-axis phase-sensitive inversion recovery LGE image in same patient. Absence of LGE in anterior wall but evidence of prior $50 \%$ transmural infarct in mid-inferior wall can be seen. Together, these images demonstrate evidence of myocardial stunning without infarction in anterior wall. 


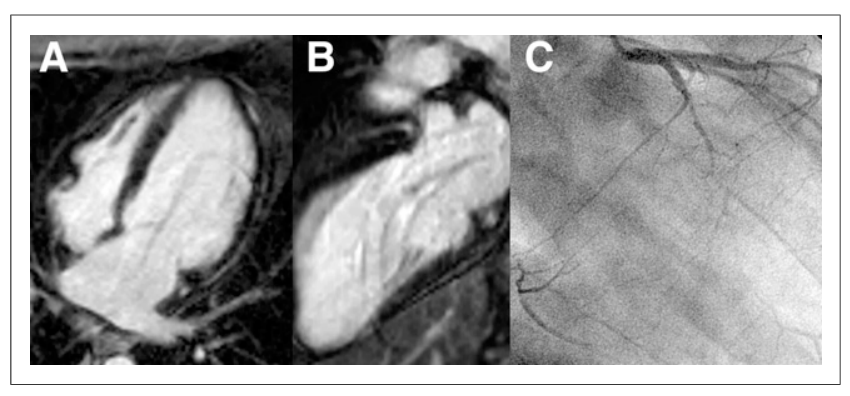

FIGURE 3. (A) Four-chamber long-axis phase-sensitive inversion recovery LGE image in patient with heart failure and EF of $20 \%$. No LGE can be seen. (B) Two-chamber long-axis phase-sensitive inversion recovery LGE image in same patient. No LGE can be seen. (C) Right anterior oblique CT angiogram demonstrating 3-vessel CAD with occluded left circumflex artery, occluded right coronary artery that fills through collateral vessels, and significant left anterior descending artery disease. Thus, this patient had severe LV dysfunction in setting of 3vessel $C A D$ without $L G E$, signifying extensive hibernating myocardium.

698 patients suggested that although LGE provided the highest sensitivity and negative predictive value $(95 \%$ and $90 \%$, respectively) for predicting functional recovery after revascularization, low-dose dobutamine cine imaging offered the highest specificity and positive predictive value (91\% and 93\%, respectively) (33).

Cardiac resynchronization therapy (CRT) with biventricular pacing has emerged as an important part of the therapeutic armamentarium for heart failure. Several studies have demonstrated that scar in the posterolateral wall is associated with a lower rate of response to CRT because the LV lead is usually placed in the lateral cardiac veins and may not appropriately capture scarred myocardium. Bleeker et al. demonstrated that, among 14 of 50 patients with greater than $50 \%$ transmural posterolateral scar, only $2(14 \%)$ responded to CRT, whereas $81 \%$ of the remainder responded (34). Another study, involving 23 patients, showed that LV scarring of greater than

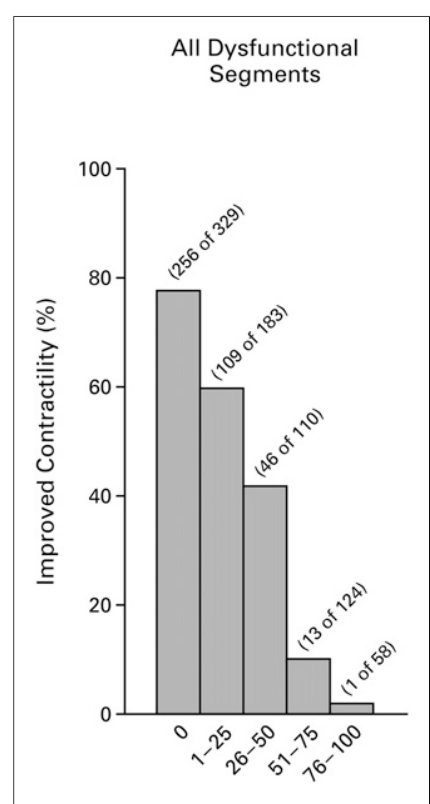

FIGURE 4. Inverse relationship between transmurality of LGE on $x$-axis and likelihood of improved function after revascularization. (Adapted with permission of (29).) addition to other, well-known risk factors, including EF and age (38).

\section{NONISCHEMIC CARDIOMYOPATHIES}

Tissue characterization by CMR is quite useful for differentiating the etiologies of nonischemic cardiomyopathies (Fig. 5). LGE can differentiate infiltrative cardiomyopathies (39), and T1 mapping can identify certain hypertrophic and infiltrative cardiomyopathies that have characteristically high native $\mathrm{T} 1$ values (40). The finding of LGE has prognostic implications for nonischemic cardiomyopathies as well as for ischemic cardiomyopathies. In a recent metaanalysis of 9 studies involving 1,488 patients who had nonischemic cardiomyopathy and were monitored for an average of $30 \mathrm{mo}$, the presence of LGE was noted in $38 \%$ of the patients and was associated with odds ratios of 3.3 for mortality and 5.3 for sudden cardiac death (SCD) or aborted SCD (41).

\section{Dilated Cardiomyopathy}

Most patients who have heart failure without obstructive CAD have no evidence of LGE (24). A minority of patients (13\%) have LGE in an infarct pattern and may be misclassified as having nonischemic cardiomyopathy (24). Approximately one-fourth of patients with DCM have evidence of midwall fibrosis (Fig. 6) (24). This likely represents the chronic healing phase of myocarditis. In a study of 472 patients who had DCM and were monitored for $5.3 \mathrm{y}, 142$ patients with midwall fibrosis had hazard ratios of 3.0 for all-cause mortality and 5.2 for a composite endpoint of SCD and aborted SCD (42). A study of 65 patients who had dilated nonischemic cardiomyopathy with an EF of less than or equal to $35 \%$ showed that the presence of LGE was associated with an 8-fold increase in heart failure, appropriate implanted cardiac device (ICD) firing, and cardiac death (43). The presence of midwall fibrosis is also an independent predictor of mortality and morbidity of patients who have DCM and are undergoing CRT (44). In fact, in that study of 97 patients with DCM and 161 patients with ischemic cardiomyopathy, the patients who had DCM with midwall fibrosis had outcomes similar to those of the patients with ischemic disease (44). Thus, as with ischemic cardiomyopathy, the presence of fibrosis or scar is a marker of an adverse outcome and a poorer response to device therapy.

\section{Myocarditis}

A typical patient with acute myocarditis has chest pain, troponin elevation, and normal coronary arteries. The finding of LGE in the midwall and subepicardium of the LV is characteristic of viral myocarditis and has been validated against histology (45). Older studies suggested that early postcontrast T1-weighted enhancement of the myocardium could be a marker of inflammation in myocarditis (46). In addition, T2-weighted imaging demonstrating myocardial edema can be a diagnostic sign. The Lake Louise Consortium in 2009 suggested that basing diagnosis on positive results from 2 of 3 techniques (early enhancement ratio, LGE, or increased T2 signal) may be an ideal approach (47).

However, the early enhancement ratio has lost favor because of a lack of reproducibility, and T2-weighted imaging has been replaced by more quantitative T2 mapping. In addition, T1 mapping, both native and with contrast for measurement of the extracellular volume, is also being used for diagnosis. Ferreira et al. studied 50 patients with suspected acute myocarditis and 45 age-matched controls and showed that the patients had higher T2 signal intensity ratios and native myocardial $\mathrm{T} 1$ values than the controls (48). In fact, a T1 cutoff of 


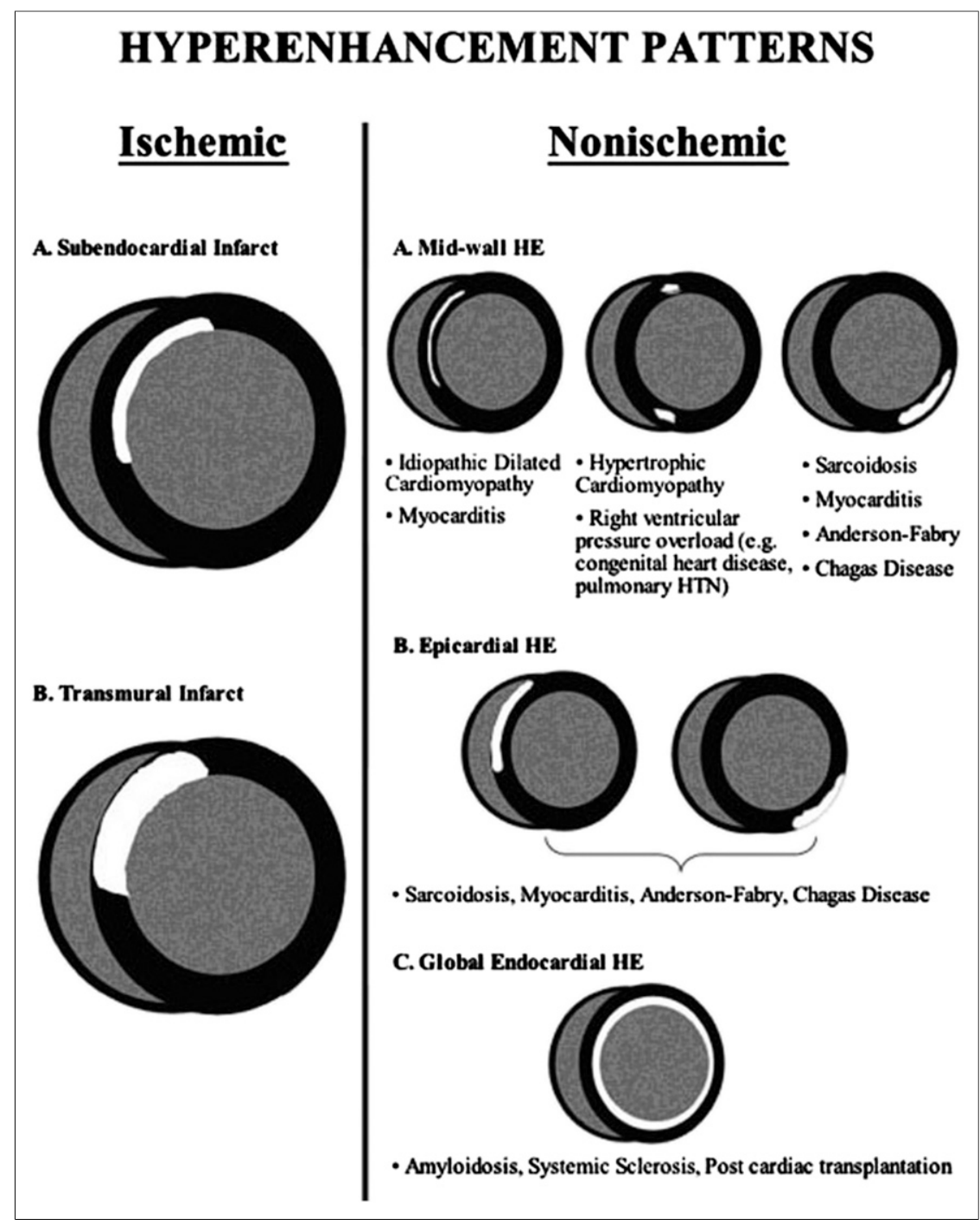

FIGURE 5. Patterns of LGE seen in ischemic and nonischemic cardiomyopathies. HE = hyperenhancement of LGE; HTN = hypertension. (Reprinted with permission of (75).) with an increased risk of cardiovascular events. Bruder et al. monitored 243 patients with HCM for $3 \mathrm{y}$ for all-cause mortality and cardiac mortality (51). LGE was seen in $67 \%$ of the patients, and the presence of LGE was associated with odds ratios of 5.5 for all-cause mortality and 8.0 for cardiac mortality. O'Hanlon et al. monitored 217 patients for $3 \mathrm{y}$ for a composite endpoint including cardiovascular death, unplanned cardiovascular admission, sustained ventricular tachycardia or ventricular fibrillation, or appropriate ICD discharge (52). Sixty-three percent of the patients had LGE, and the presence of LGE was associated with an odds ratio of 3.4 for the primary endpoint. A metaanalysis of data pooled from 1,063 patients monitored for an average of $3.1 \mathrm{y}$ in 4 studies, including the latter 2, demonstrated that the presence of LGE was associated with odds ratios of 2.9 for cardiac death, 5.7 for heart failure death, and 4.5 for all-cause mortality but only a trend for SCD (53). These studies treated LGE in a binary fashion, but it is likely that the extent of LGE adds discriminatory value.

A recent study of 1,293 patients monitored for $3.3 \mathrm{y}$ showed that LGE of greater than or equal to $15 \%$ of the LV mass was associated with a 2 -fold increase in SCD event risk (54). In addition to the amount, the location or pattern of LGE may be more predictive of adverse outcome than the presence of LGE alone. The ongoing National Institutes of Health-funded natural history study, "HCMR: Novel Predictors of Prognosis in Hypertrophic Cardiomyopathy" (NCT01915615), involving CMR, genetics, and biomarker evaluation of 2,750 $990 \mathrm{~ms}$ at $1.5 \mathrm{~T}$ demonstrated sensitivity, specificity, and diagnostic accuracy of $90 \%, 91 \%$, and $91 \%$, respectively. A combination of these techniques may be the best way to accurately identify acute myocarditis. In a recent study of 104 patients and 21 controls, a stepwise approach involving LGE and a myocardial extracellular volume of greater than $27 \%$ showed an overall accuracy of $90 \%$ (49). In another study, involving 61 patients with acute myocarditis and 67 patients with chronic myocarditis, the diagnostic accuracies for acute myocarditis were $99 \%$ for native T1, $86 \%$ for LGE alone, and $72 \%$ for increased T2 signal (50). For chronic myocarditis, LGE alone performed better than T1 mapping (94\% vs. $84 \%$ accuracies), but the combination of the 2 techniques performed even better, with a $98 \%$ overall accuracy.

\section{HCM}

Approximately two-thirds of patients with HCM may have LGE with a characteristic pattern of patchy involvement, particularly at RV septal insertion sites and in walls with the greatest hypertrophy. Several studies have examined the relationship between the presence of LGE and outcome in patients with HCM. Two recent studies, although underpowered, showed that LGE was associated patients with HCM, is likely to offer further insight in this regard.

\section{Amyloidosis}

In addition to the classic findings of thick LV walls, valves, and interatrial septum and the presence of a pericardial effusion in cardiac amyloidosis, the existence of amyloid protein in the myocardial interstitium is associated with characteristic patterns of LGE due to abnormal gadolinium kinetics in the infiltrated myocardium (55). Validation against endomyocardial biopsy was performed in 33 patients with diastolic dysfunction and features suggesting the presence of amyloid protein (56), and the pattern of circumferential subendocardial LGE had a sensitivity of $80 \%$ and a specificity of $94 \%$ (Fig. 7). When the volume of distribution of gadolinium was quantified with serial measures of $\mathrm{T} 1$ in the myocardium after gadolinium contrast infusion, it was found to be markedly larger than that in healthy controls (57). In addition, the "native" T1 of the myocardium was significantly longer in patients with amyloidosis than in controls (57) or in patients with left ventricular hypertrophy due to aortic stenosis (58). 


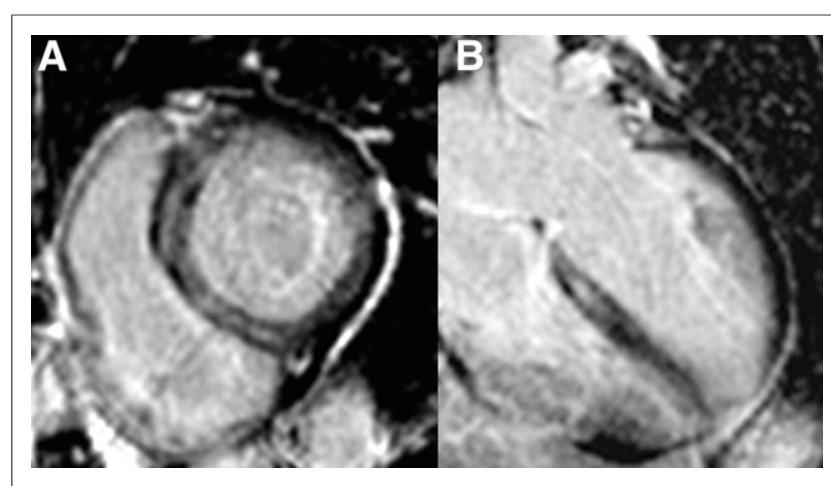

FIGURE 6. (A) Basal short-axis phase-sensitive inversion recovery LGE image in patient with heart failure, global LV systolic dysfunction, and EF of $25 \%$. Midwall stripe of LGE can be seen in septum. (B) Fourchamber long-axis phase-sensitive inversion recovery LGE image in same patient, again showing midseptal stripe of LGE.

CMR is increasingly being used to discriminate light-chain (AL) amyloidosis from the transthyretin (ATTR) form of the disease. Fontana et al. studied native T1 mapping in 85 patients with ATTR disease, 79 patients with AL disease, 52 healthy subjects, and 46 patients with $\mathrm{HCM}(59)$. AL disease showed the highest native T1, followed by ATTR disease, although the areas under the receiver operating characteristic curves were similar for both forms of amyloidosis versus HCM (0.84). The potential utility of native T1 is critically important, as many patients with amyloidosis have concomitant renal dysfunction and are not candidates for receiving gadolinium because of concerns about nephrogenic systemic fibrosis. In another study, LGE findings in 46 patients with biopsy-proven AL amyloidosis and 51 patients with ATTR amyloidosis were compared (60). LGE was much more extensive in patients with ATTR amyloidosis, with 90\% demonstrating transmural LGE, compared with only $37 \%$ of patients with AL amyloidosis. These investigators developed an LGE scoring system that differentiated the 2 types with $87 \%$ sensitivity and $96 \%$ specificity.

\section{Sarcoidosis}

CMR can readily identify characteristic features of cardiac sarcoidosis, including biventricular dilation and dysfunction. The pattern of LGE seen with cardiac sarcoid is variable; a classic pattern of midwall or epicardial LGE can be seen, but subendocardial or transmural enhancement in almost any distribution is also possible. A study of CMR involving 58 patients with biopsy-proven pulmonary sarcoid found 19 patients with evidence of LGE, mostly in the basal and lateral myocardium; this prevalence was higher than that identified by standard Japanese Ministry of Health guidelines (61). In another study, involving 81 patients who had extracardiac sarcoidosis and were monitored for an average of $21 \mathrm{mo}$, a 2-fold-higher rate of cardiac involvement was demonstrated by CMR than by Japanese Ministry of Health guidelines (62). Patients with LGE had a 9-fold-higher incidence of adverse events than those without LGE. In addition, T2 mapping may have a role in delineating the activity of sarcoidosis. In a study of 28 patients, regions of LGE showed decreased T2; this finding may have reflected an inactive phase of the disease (63).

\section{Arrhythmogenic Right Ventricular Cardiomyopathy}

CMR is the gold standard for the diagnosis of arrhythmogenic RV cardiomyopathy. Diagnostic findings in this disease include
RV dilatation and global or regional dysfunction, including focal RV systolic bulging or aneurysm, and are carefully defined to meet Task Force criteria (64). In certain cases, LGE of the RV free wall can be identified (65), although it can be difficult to identify because of the thin RV wall in this disorder.

\section{Noncompaction Cardiomyopathy}

Noncompaction cardiomyopathy is defined on CMR as a ratio of noncompacted myocardium to compacted myocardium of 2.3 at the end of diastole (66). This ratio should be measured at a distance from the true apex. Newer definitions for a CMR diagnosis are frequently entertained (67), although the ratio of noncompacted myocardium to compacted myocardium of 2.3 remains the standard at present. Additional CMR findings in this disorder may include LV systolic dysfunction and LV thrombus.

\section{Other Nonischemic Cardiomyopathies}

CMR is also able to diagnose a variety of other nonischemic cardiomyopathies, including takotsubo cardiomyopathy, demonstrating myocardial edema without LGE (68); iron-overload cardiomyopathy (with T2* mapping) (69); Anderson-Fabry disease, with characteristic findings of basal and lateral LGE (70); and Chagas disease, with various LGE findings (71).

\section{LIMITATIONS OF CMR IN CARDIOMYOPATHIES}

There are several limitations to the use of CMR for cardiomyopathies. For one, many patients with heart failure and a reduced EF receive ICDs, and some with more severe heart failure refractory to medical therapy receive CRT devices. At present, both ICDs and CRT devices are contraindicated for performing CMR. This contraindication limits the use of CMR in patients with more advanced heart failure. However, there is growing evidence of the safety of many of these devices under stringent conditions in MR scanners (72). In addition, with the growth in the implantation of MR-conditional pacemaker systems, there is recognition of the ability to perform cardiac imaging safely in patients with these devices (73). Another limitation is that patients with stage 4 or 5 chronic kidney disease cannot receive gadolinium because of concerns about the potential of causing nephrogenic systemic fibrosis in this population (74). Patients who have active heart failure and are unable to lie flat are also not candidates for CMR.

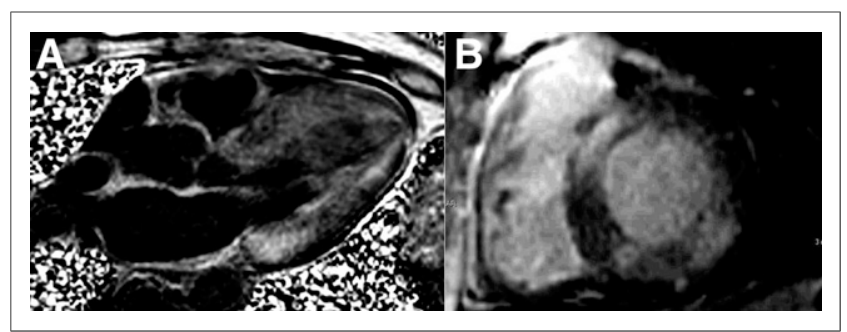

FIGURE 7. (A) Four-chamber long-axis phase-sensitive inversion recovery LGE image in patient with heart failure and EF of $30 \%$. Diffuse subendocardial LGE and signal nulling in blood pool, all characteristic of amyloidosis (most likely ATTR variety), can be seen. (B) Basal short-axis phase-sensitive inversion recovery LGE image in different patient with heart failure, global LV systolic dysfunction, and EF of $25 \%$. In this patient, LGE is patchy and distribution is noncoronary-more consistent with $\mathrm{AL}$ amyloidosis-which was subsequently diagnosed by fat pad biopsy. 


\section{CONCLUSION}

CMR is an extremely useful imaging technique for cardiomyopathies. CMR is the gold standard for the measurement of cardiac chamber size and function. With tissue characterization by LGE as well as T1 and T2 mapping, the underlying etiology of heart failure can be readily established. In addition, the presence and extent of LGE in cardiomyopathies are associated with adverse cardiovascular outcomes and poor responses to standard medical and interventional therapies. Thus, CMR has a major role to play in determining diagnosis and assessing prognosis for patients with cardiomyopathies.

\section{DISCLOSURE}

The costs of publication of this article were defrayed in part by the payment of page charges. Therefore, and solely to indicate this fact, this article is hereby marked "advertisement" in accordance with 18 USC section 1734. Christopher M. Kramer is supported in part by R01 HL075792 and U01HL117006-01A1; receives equipment support from Siemens Healthcare; and has consulted for Bristol-Meyers Squibb, Merck, Myokardia, and Heart Metabolomics. No other potential conflict of interest relevant to this article was reported.

\section{REFERENCES}

1. Miller S, Simonetti OP, Carr J, Kramer U, Finn JP. MR imaging of the heart with cine true fast imaging with steady-state precession: influence of spatial and temporal resolutions on left ventricular functional parameters. Radiology. 2002;223:263-269.

2. Isbell DC, Kramer CM. Cardiovascular magnetic resonance: structure, function, perfusion, and viability. J Nucl Cardiol. 2005;12:324-336.

3. Grothues F, Smith GC, Moon JCC, et al. Comparison of interstudy reproducibility of cardiovascular magnetic resonance with two-dimensional echocardiography in normal subjects and in patients with heart failure or left ventricular hypertrophy. Am J Cardiol. 2002;90:29-34.

4. Bellenger NG, Davies LC, Francis JM, Coats AJS, Pennell DJ. Reduction in sample size for studies of remodeling in heart failure by the use of cardiovascular magnetic resonance. J Cardiovasc Magn Reson. 2000;2:271-278.

5. Zerhouni EA, Parish DM, Rogers WJ, Yang A, Shapiro EP. Human heart: tagging with MR imaging - a new method for noninvasive assessment of myocardial motion. Radiology. 1988;169:59-63.

6. Kim D, Gilson WD, Kramer CM, Epstein FH. Myocardial tissue tracking with two-dimensional cine displacement-encoded MR imaging: development and initial evaluation. Radiology. 2004;230:862-871.

7. Kramer CM, Rogers WJ, Theobald TM, Power TP, Petruolo S, Reichek N. Remote noninfarcted region dysfunction soon after first anterior myocardial infarction: a magnetic resonance tagging study. Circulation. 1996;94:660-666.

8. Kramer CM, Reichek N, Ferrari VA, Theobald T, Dawson J, Axel L. Regional heterogeneity of function in hypertrophic cardiomyopathy. Circulation. 1994;90: 186-194.

9. Rehr RB, Peshock RM, Malloy CR, et al. Improved in vivo magnetic resonance imaging of acute myocardial infarction after intravenous paramagnetic contrast agent administration. Am J Cardiol. 1986;57:864-868.

10. Peshock RM, Malloy CR, Buja LM, Nunnally RL, Parkey RW, Willerson JT. Magnetic resonance imaging of acute myocardial infarction: gadolinium diethylenetriamine pentaacetic acid as a marker of reperfusion. Circulation. 1986;74: 1434-1440.

11. de Roos A, Doornbos J, van der Wall EE, Van Voorthuisen AE. MR imaging of acute myocardial infarction: value of Gd-DTPA. AJR. 1988;150:531-534.

12. Simonetti OP, Kim RJ, Fieno DS, et al. An improved MR imaging technique for the visualization of myocardial infarction. Radiology. 2001;218:215-223.

13. Kim RJ, Fieno DS, Parrish TB, et al. Relationship of MRI delayed contrast enhancement to irreversible injury, infarct age, and contractile function. Circulation. 1999;100:1992-2002.

14. Rehwald WG, Fieno DS, Chen EL, Kim RJ, Judd RM. Myocardial magnetic resonance imaging contrast agent concentrations after reversible and irreversible ischemic injury. Circulation. 2002;105:224-229.

15. Fieno DS, Kim RJ, Chen EL, Lomasney JW, Klocke FJ, Judd RM. Contrastenhanced magnetic resonance imaging of myocardium at risk: distinction between reversible and irreversible injury throughout infarct healing. J Am Coll Cardiol. 2000;36:1985-1991.

16. Abdel-Aty H, Cocker M, Meek C, Tyberg JV, Friedrich MG. Edema as a very early marker for acute myocardial ischemia: a cardiovascular magnetic resonance study. J Am Coll Cardiol. 2009;53:1194-1201.

17. Aletras AH, Tilak GS, Natanzon A, et al. Retrospective determination of the area at risk for reperfused acute myocardial infarction with T2-weighted cardiac magnetic resonance imaging: histopathological and displacement encoding with stimulated echoes (DENSE) functional validations. Circulation. 2006;113:18651870 .

18. Abdel-Aty H, Boye P, Zagrosek A, et al. Diagnostic performance of cardiovascular magnetic resonance in patients with suspected acute myocarditis: comparison of different approaches. J Am Coll Cardiol. 2005;45:1815-1822.

19. Giri S, Chung YC, Merchant A, et al. T2 quantification for improved detection of myocardial edema. J Cardiovasc Magn Reson. 2009;11:56.

20. Messroghli DR, Walters K, Plein S, et al. Myocardial T1 mapping: application to patients with acute and chronic myocardial infarction. Magn Reson Med. 2007;58: 34-40.

21. Piechnik SK, Ferreira VM, Dall'Armellina E, et al. Shortened Modified LookLocker Inversion recovery (ShMOLLI) for clinical myocardial T1-mapping at 1.5 and 3 T within a 9 heartbeat breathhold. J Cardiovasc Magn Reson. 2010;12: 69.

22. Moon JC, Messroghli DR, Kellman P, et al. Myocardial T1 mapping and extracellular volume quantification: a Society for Cardiovascular Magnetic Resonance (SCMR) and CMR Working Group of the European Society of Cardiology consensus statement. J Cardiovasc Magn Reson. 2013;15:92.

23. Soriano CJ, Ridocci F, Estornell J, Jimenez J, Martinez V, De Velasco JA. Noninvasive diagnosis of coronary artery disease in patients with heart failure and systolic dysfunction of uncertain etiology, using late gadolinium-enhanced cardiovascular magnetic resonance. J Am Coll Cardiol. 2005;45:743-748.

24. McCrohon JA, Moon JC, Prasad SK, et al. Differentiation of heart failure related to dilated cardiomyopathy and coronary artery disease using gadolinium-enhanced cardiovascular magnetic resonance. Circulation. 2003;108:54-59.

25. le Polain de Waroux JB, Pouleur AC, Goffinet C, Pasquet A, Vanoverschelde JL, Gerber BL. Combined coronary and late-enhanced multidetector-computed tomography for delineation of the etiology of left ventricular dysfunction: comparison with coronary angiography and contrast-enhanced cardiac magnetic resonance imaging. Eur Heart J. 2008;29:2544-2551.

26. Bello D, Shah DJ, Farah GM, et al. Gadolinium cardiovascular magnetic resonance predicts reversible myocardial dysfunction and remodeling in patients with heart failure undergoing beta-blocker therapy. Circulation. 2003;108:1945-1953.

27. Baer FM, Voth E, Schneider CA, Theissen P, Schicha H, Sechtem U. Comparison of low-dose dobutamine-gradient-echo magnetic resonance imaging and positron emission tomography with $\left[{ }^{18} \mathrm{~F}\right]$ fluorodeoxyglucose in patients with chronic coronary artery disease: a functional and morphological approach to the detection of residual myocardial viability. Circulation. 1995;91:1006-1015.

28. Shah DJ, Kim HW, James O. Prevalence of regional myocardial thinning and relationship with myocardial scarring in patients with coronary artery disease. JAMA. 2013;309:909-918.

29. Kim RJ, Wu E, Rafael A, et al. The use of contrast-enhanced magnetic resonance imaging to identify reversible myocardial dysfunction. N Engl J Med. 2000;343: 1445-1453.

30. Selvanayagam JB, Kardos A, Francis JM, et al. Value of delayed-enhancement cardiovascular magnetic resonance imaging in predicting myocardial viability after surgical revascularization. Circulation. 2004;110:1535-1541.

31. Bove CM, DiMaria JM, Voros S, Conaway MR, Kramer CM. Dobutamine response and myocardial infarct transmurality: functional improvement after coronary artery bypass grafting_initial experience. Radiology. 2006;240:835-841.

32. Wellnhofer E, Olariu A, Klein C, et al. Magnetic resonance low-dose dobutamine test is superior to SCAR quantification for the prediction of functional recovery. Circulation. 2004;109:2172-2174.

33. Romero J, Xue X, Gonzalez W, Garcia MJ. CMR imaging assessing viability in patients with chronic ventricular dysfunction due to coronary artery disease: a meta-analysis of prospective trials. JACC Cardiovasc Imaging. 2012;5: 494-508.

34. Bleeker GB, Kaandorp TAM, Lamb HJ, et al. Effect of posterolateral scar tissue on clinical and echocardiographic improvement after cardiac resynchronization therapy. Circulation. 2006;113:969-976.

35. White JA, Yee R, Yuan X, et al. Delayed enhancement magnetic resonance imaging predicts response to cardiac resynchronization therapy in patients with intraventricular dyssynchrony. J Am Coll Cardiol. 2006;48:1953-1960.

36. Bilchick KC, Kuruvilla S, Hamirani YS, et al. Impact of mechanical activation, scar, and electrical timing on cardiac resynchronization therapy response and clinical outcomes. J Am Coll Cardiol. 2014;63:1657-1666. 
37. Kwong RY, Chan AK, Brown KA, et al. Impact of unrecognized myocardial scar detected by cardiac magnetic resonance imaging on event-free survival in patients presenting with signs or symptoms of coronary artery disease. Circulation. 2006;113:2733-2743.

38. Cheong BYC, Muthupillai R, Wilson JM, et al. Prognostic significance of delayed-enhancement magnetic resonance imaging: survival of 857 patients with and without left ventricular dysfunction. Circulation. 2009;120:2069-2076.

39. Karamitsos TD, Francis JM, Neubauer S. The current and emerging role of cardiovascular magnetic resonance in the diagnosis of nonischemic cardiomyopathies. Prog Cardiovasc Dis. 2011;54:253-265.

40. Dass S, Suttie JJ, Piechnik SK, et al. Myocardial tissue characterization using magnetic resonance noncontrast T1 mapping in hypertrophic and dilated cardiomyopathy. Circ Cardiovasc Imaging. 2012;5:726-733.

41. Kuruvilla S, Adenaw N, Katwal AB, Lipinski MJ, Kramer CM, Salerno M. Late gadolinium enhancement on cardiac magnetic resonance predicts adverse cardiovascular outcomes in nonischemic cardiomyopathy: a systematic review and meta-analysis. Circ Cardiovasc Imaging. 2014;7:250-258.

42. Gulati A, Jabbour A, Ismail TF. Association of fibrosis with mortality and sudden cardiac death in patients with nonischemic dilated cardiomyopathy. JAMA. 2013;309:896-908.

43. Wu KC, Weiss RG, Thiemann DR, et al. Late gadolinium enhancement by cardiovascular magnetic resonance heralds an adverse prognosis in nonischemic cardiomyopathy. J Am Coll Cardiol. 2008;51:2414-2421.

44. Leyva F, Taylor RJ, Foley PWX, et al. Left ventricular midwall fibrosis as a predictor of mortality and morbidity after cardiac resynchronization therapy in patients with nonischemic cardiomyopathy. J Am Coll Cardiol. 2012;60: 1659-1667.

45. Mahrholdt H, Goedecke C, Wagner A, et al. Cardiovascular magnetic resonance assessment of human myocarditis: a comparison to histology and molecular pathology. Circulation. 2004;109:1250-1258.

46. Friedrich MG, Strohm O, Schulz-Menger J, Marciniak H, Luft FC, Dietz R. Contrast media-enhanced magnetic resonance imaging visualizes myocardial changes in the course of viral myocarditis. Circulation. 1998;97:1802-1809.

47. Friedrich MG, Sechtem U, Schulz-Menger J, et al. Cardiovascular magnetic resonance in myocarditis: a JACC white paper. J Am Coll Cardiol. 2009;53: 1475-1487.

48. Ferreira VM, Piechnik SK, Dall'Armellina E, et al. T1 mapping for the diagnosis of acute myocarditis using CMR: comparison to T2-weighted and late gadolinium enhanced imaging. JACC Cardiovasc Imaging. 2013;6:1048-1058.

49. Radunski UK, Lund GK, Stehning C, et al. CMR in patients with severe myocarditis: diagnostic value of quantitative tissue markers including extracellular volume imaging. JACC Cardiovasc Imaging. 2014;7:667-675.

50. Hinojar R, Foote L, Arroyo Ucar E, et al. Native T1 in discrimination of acute and convalescent stages in patients with clinical diagnosis of myocarditis: a proposed diagnostic algorithm using CMR. JACC Cardiovasc Imaging. 2015; 8:37-46.

51. Bruder O, Wagner A, Jensen CJ, et al. Myocardial scar visualized by cardiovascular magnetic resonance imaging predicts major adverse events in patients with hypertrophic cardiomyopathy. J Am Coll Cardiol. 2010;56:875-887.

52. O'Hanlon R, Grasso A, Roughton M, et al. Prognostic significance of myocardial fibrosis in hypertrophic cardiomyopathy. J Am Coll Cardiol. 2010;56:867-874.

53. Green JJ, Berger J, Kramer CM, Salerno M. Prognostic value of cardiac magnetic resonance late gadolinium enhancement in clinical outcomes for hypertrophic cardiomyopathy. JACC Cardiovasc Imaging. 2012;5:370-377.

54. Chan RH, Maron BJ, Olivotto I, et al. Prognostic value of quantitative contrastenhanced cardiovascular magnetic resonance for the evaluation of sudden death risk in patients with hypertrophic cardiomyopathy. Circulation. 2014; 130:484-495.
55. Maceira AM, Joshi J, Prasad SK, et al. Cardiovascular magnetic resonance in cardiac amyloidosis. Circulation. 2005;111:186-193.

56. Vogelsberg H, Mahrholdt H, Deluigi CC, et al. Cardiovascular magnetic resonance in clinically suspected cardiac amyloidosis: noninvasive imaging compared to endomyocardial biopsy. J Am Coll Cardiol. 2008;51:1022-1030.

57. Brooks J, Kramer CM, Salerno M. Markedly increased volume of distribution of gadolinium in cardiac amyloidosis demonstrated by T1 mapping. J Magn Reson Imaging. 2013;38:1591-1595.

58. Karamitsos TD, Piechnik SK, Banypersad SM, et al. Noncontrast T1 mapping for the diagnosis of cardiac amyloidosis. JACC Cardiovasc Imaging. 2013;6: 488-497.

59. Fontana M, Banypersad SM, Treibel TA, et al. Native myocardial T1 mapping in transthyretin amyloidosis. JACC Cardiovasc Imaging. 2014;7:157-165.

60. Dungu JN, Valencia O, Pinney JH, et al. CMR-based differentiation of AL and ATTR cardiac amyloidosis. JACC Cardiovasc Imaging. 2014;7:133-142.

61. Smedema JP, Snoep G, van Kroonenburgh MP, et al. Evaluation of the accuracy of gadolinium-enhanced cardiovascular magnetic resonance in the diagnosis of cardiac sarcoidosis. J Am Coll Cardiol. 2005;45:1683-1690.

62. Patel MR, Cawley PJ, Heitner JF, et al. Detection of myocardial damage in patients with sarcoidosis. Circulation. 2009;120:1969-1977.

63. Yang Y, Safka K, Graham JJ, et al. Correlation of late gadolinium enhancement MRI and quantitative T2 measurement in cardiac sarcoidosis. J Magn Reson Imaging. 2014;39:609-616.

64. Marcus FI, McKenna WJ, Sherrill D, et al. Diagnosis of arrhythmogenic right ventricular cardiomyopathy/dysplasia: proposed modification of the task force criteria. Circulation. 2010;121:1533-1541.

65. Tandri H, Saranathan M, Rodriguez ER, et al. Noninvasive detection of myocardial fibrosis in arrhythmogenic right ventricular cardiomyopathy using delayedenhancement magnetic resonance imaging. J Am Coll Cardiol. 2005;45:98-103.

66. Petersen SE, Selvanayagam JB, Wiesmann F, et al. Left ventricular non-compaction: insights from cardiovascular magnetic resonance imaging. $\mathrm{J} \mathrm{Am} \mathrm{Coll} \mathrm{Cardiol.}$ 2005;46:101-105.

67. Stacey RB, Andersen MM, St. Clair M, Hundley WG, Thohan V. Comparison of systolic and diastolic criteria for isolated LV noncompaction in CMR. JACC Cardiovasc Imaging. 2013;6:931-940.

68. Eitel I, von Knobelsdorff-Brenkenhoff F, Bernhardt P. Clinical characteristics and cardiovascular magnetic resonance findings in stress (takotsubo) cardiomyopathy. JAMA. 2011;306:277-286.

69. Kirk P, Roughton M, Porter JB, et al. Cardiac T2* magnetic resonance for prediction of cardiac complications in thalassemia major. Circulation. 2009;120: 1961-1968.

70. Moon JCC, Sachdev B, Elkington AG, et al. Gadolinium enhanced cardiovascular magnetic resonance in Anderson-Fabry disease. Eur Heart J. 2003;24: 2151-2155.

71. Rochitte CE, Oliveira PF, Andrade JM, et al. Myocardial delayed enhancement by magnetic resonance imaging in patients with Chagas' disease: a marker of disease severity. J Am Coll Cardiol. 2005;46:1553-1558.

72. Nazarian S, Hansford R, Roguin A, et al. A prospective evaluation of a protocol for magnetic resonance imaging of patients with implanted cardiac devices. Ann Intern Med. 2011;155:415-424.

73. Wollmann CG, Thudt K, Kaiser B, Salomonowitz E, Mayr H, Globits S. Safe performance of magnetic resonance of the heart in patients with magnetic resonance conditional pacemaker systems: the safety issue of the ESTIMATE study. J Cardiovasc Magn Reson. 2014;16:30.

74. Kaewlai R, Abujudeh H. Nephrogenic systemic fibrosis. AJR. 2012;199: W17-W23.

75. Edelman RR, Hesselink JR, Zlatkin MB, Crues JV III. Clinical Magnetic Resonance Imaging. New York, NY: Elsevier; 2005. 